\title{
Aneurysmal Bone Cyst of the Distal Femoral Metaphysis in a Four-year-old Female Patient Presenting with a Pathologic Fracture: A Case Report
}

\author{
James Dorosh $^{1}$, Parth Vyas ${ }^{1}$ \\ 1. Orthopedic Oncology, Sanford Medical Center, Fargo, USA
}

$\square$ Corresponding author: James Dorosh, james.m.dorosh@dmu.edu

Disclosures can be found in Additional Information at the end of the article

\section{Abstract}

Aneurysmal bone cysts (ABCs) are benign osteolytic vascular lesions that are capable of aggressive local expansion and bone destruction. These tumors are most common in adolescent patients and constitute approximately $9 \%$ of benign tumors. ABCs can present a diagnostic challenge, as they share several histological and radiographic characteristics with more aggressive lesions, including giant cell tumors and malignant telangiectatic osteosarcomas. The management of $\mathrm{ABCs}$ is diverse, but the most common approach includes lesion curettage with bone grafting. Here, we present the case of a large, central $\mathrm{ABC}$ of the distal femur in a young, previously healthy female who presented to the emergency room with a pathologic fracture.

Categories: Pathology, Oncology, Orthopedics

Keywords: aneurysmal bone cyst, telangiectatic osteosarcoma, orthopedic oncology, capanna criteria, bone curettage, pathological fracture

\section{Introduction}

Aneurysmal bone cysts (ABCs) are benign osteolytic vascular lesions that are capable of aggressive local expansion and bone destruction [1]. Because of this, morbidity and symptomatology tend to arise from local tissue destruction and pathologic fracture. Patients typically present during the second decade of life and ABCs represent approximately $9 \%$ of benign bone tumors [2]. Care must be taken to differentiate the diagnosis of $A B C$ from a unicameral bone cyst and telangiectatic osteosarcoma as the treatment protocol is different [3]. Tissue biopsy with histologic interpretation must be consistently employed to avoid misdiagnosis. If the lesion is amenable, curettage with bone grafting is the recommended initial approach to management [4]. Adjuvant therapies are multiple and include electrocautery, cryotherapy, intra-lesional sclerotherapy, preoperative embolization, and medical therapy with denosumab as fracture prophylaxis [4-5]. Despite appropriate management, reports of tumor recurrence range considerably, with different treatment options from as low as $5 \%$ to more than $40 \%[6]$.

Here, we will discuss the unique case of a large central Type 1 Campanacci criteria ABC of the distal femur in a young, previously healthy female who presented to the emergency room with a pathologic fracture after sustaining a fall from the standing height. This paper will also review the different radiographic types of $\mathrm{ABCs}$, discuss the radiographic and histologic differentiation from telangiectatic osteosarcoma, and discuss the recommended management. 


\section{Case Presentation}

A four-year-old girl presented to the emergency department (ED) complaining of sudden onset severe left thigh pain after sustaining a fall from the standing height while playing a game of tag. The patient's parents denied pain with ambulation prior to this episode, previous nocturnal pain, a family or patient history of cancer, fever, chills, weight loss, or any other symptoms. On physical exam, the patient was in moderate distress and screamed at any attempt to move her leg. She was unable to bear weight on the affected leg and the thigh was exquisitely tender to palpation with diffuse swelling.

X-rays demonstrated an expansile lytic lesion of the left distal femur with a pathologic fracture and a positive fallen leaf sign (Figure $1 \mathrm{~A}$ ). Magnetic resonance (MR) imaging showed a lesion measuring $3.5 \times 3.0 \mathrm{~cm}$ in the greatest axial cross-section and $5.2 \mathrm{~cm}$ in the craniocaudal extent. The lesion was centered within the metaphysis and appeared expansile with surrounding cortical thinning, without invasion of the physis. There were multiple blood-filled cystic spaces with fluid levels. There were no underlying soft tissue elements noted, suggesting this was a primary $A B C$ (Figures $1 B-1 C$ ).

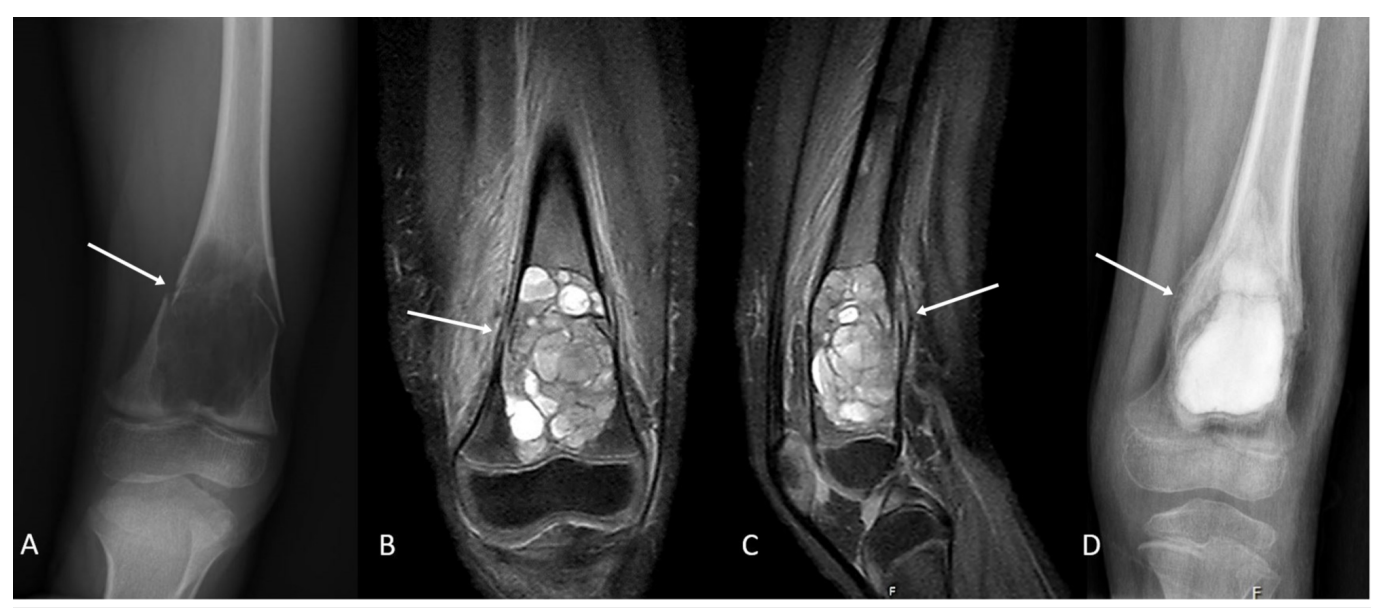

FIGURE 1: (A) Plain anteroposterior radiographic film demonstrating a left femoral distal metaphyseal expansile lytic lesion with a fallen leaf type fracture. (B,C) Coronal and sagittal magnetic resonance (MR) images demonstrating expansile lesion of the distal metaphysis of the femur. No underlying soft tissue elements are noted, suggesting the lesion is most likely a primary aneurysmal bone cyst. (D) Plain anteroposterior radiographic film demonstrating callus formation and postop changes at six weeks of follow-up.

Left femoral bone cyst curettage and bone grafting were performed. First, the femur was exposed through a $3 \mathrm{~cm}$ lateral incision of the distal thigh and a periosteal window in the bone was created. Electrocautery was used to achieve hemostasis and lesion curettage was performed. Tissue samples were analyzed as a frozen section and, once determined to be a benign lesion, the lesion was thoroughly curetted. Chemical adjuvant was withheld secondary to the close proximity of the lesion to the growth plate. A titanium nail was used to establish communication between the various cystic components of the lesion and the bony medullary 
canal. A bone void filler consisting of calcium sulfate and calcium phosphate was placed under $\mathrm{X}$-ray guidance. After confirming appropriate fracture reduction, the patient was closed and placed in a Spica cast. The formal pathological report demonstrated a cystic lesion with bland fibroblasts and myofibroblasts admixed with seams of osteoid and scattered multinucleated giant cells without significant cellular atypia suggestive of ABC (Figure 2).

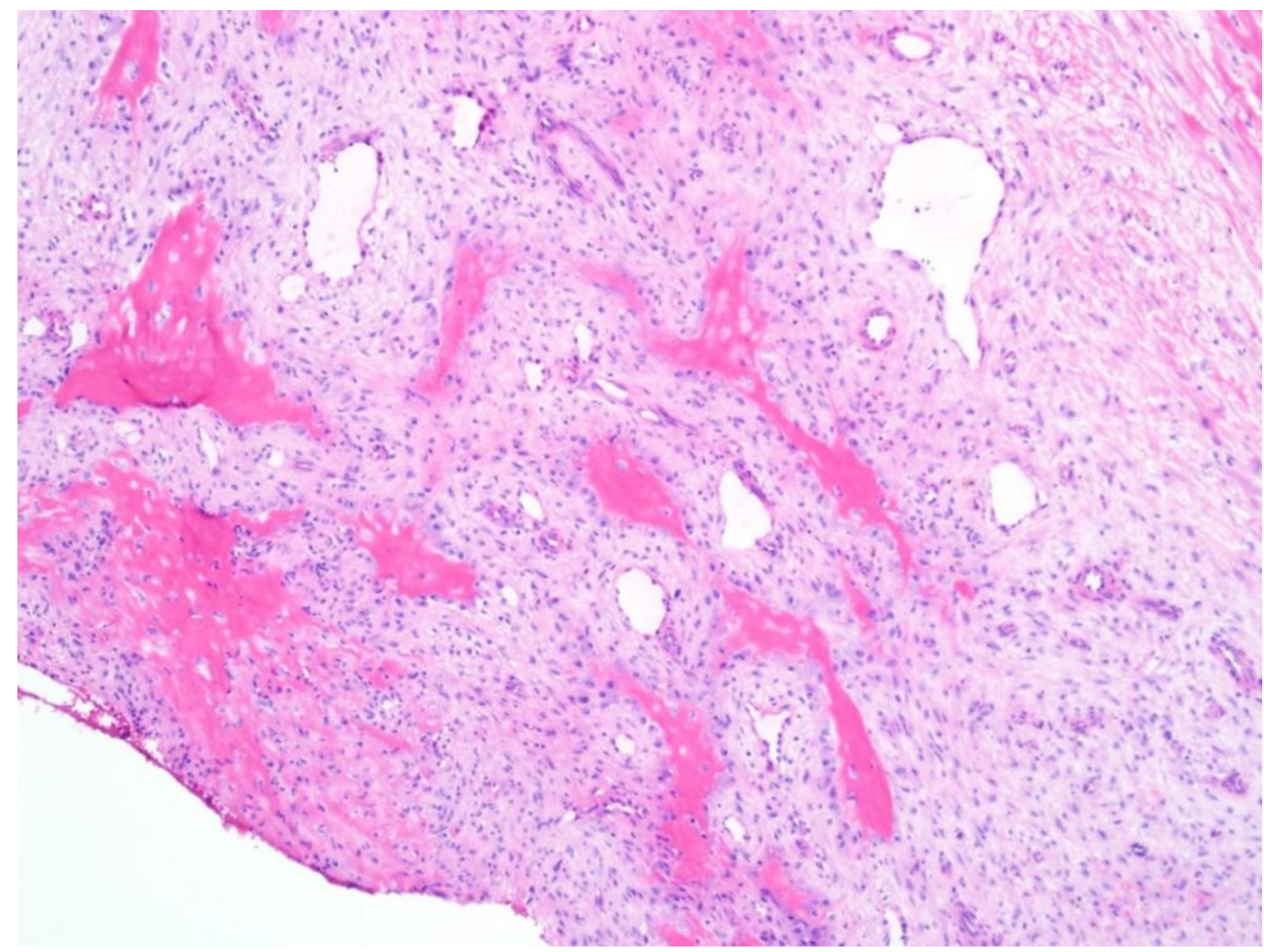

FIGURE 2: Stained histologic section demonstrating a cystic lesion composed of fibroblasts and myofibroblasts admixed with seams of osteoid and scattered multinucleated giant cells without significant cellular atypia consistent with an aneurysmal bone cyst.

At the six-week follow-up appointment, X-rays demonstrated interval consolidation of the bone graft with callus formation at the fracture site (Figure $1 D$ ). There was no sign of lesion recurrence and the patient was able to return to full preoperative weight-bearing activities.

\section{Discussion}

The incidence of ABCs is approximately 0.14 per 100,000 persons each year, and they typically present during a patient's adolescent years [3]. This case represents a minority of patients that can present at an earlier date. The skeletal location of distal femoral metaphysis is an important weight-bearing location where the patient's first presentation is commonly following trauma and fracture. This presentation can complicate treatment.

In 1986, a radiographic classification system of ABCs, along with the recommended management and expected outcomes, was presented by Campanacci et al. These ABC grading criteria are often referred to in an abbreviated manner simply as the "Campanacci criteria," as 


\section{Cureus}

shown in Table 1. These criteria distinguish amongst five unique $\mathrm{ABC}$ types based on their radiographic appearance. Briefly, Type 1 cysts are centrally arising and can spread to the cortical edge of bones, sometimes enlarging the total diameter of the bone. They are typically located in the metaphysis or metadiaphysis of long bones. Type 2 cysts are centrally located and replace the entirety of the involved boney segment within which they occur. Type 3 cysts arise eccentrically, with little to no cortical involvement. Type 4 cysts are subperiosteal, usually arising at the diaphysis of long bones, and lift the periosteum circumferentially as they expand. Finally, Type 5 cysts are also subperiosteal and elevate the periosteum circumferentially, as in Type 4; however, they also destroy the adjacent cortical and cancellous bone as they enlarge [7].

\begin{tabular}{|c|c|c|c|}
\hline Type & Osteologic Site & Skeletal Site & Radiographic Appearance \\
\hline 1 & central & $\begin{array}{l}\text { long bone metaphysis and } \\
\text { metadiaphysis }\end{array}$ & cystic lucency with cortical attenuation \\
\hline 2 & central expansive & $\begin{array}{l}\text { long bone metaphysis and } \\
\text { metadiaphysis }\end{array}$ & $\begin{array}{l}\text { cystic lucency with severe cortical } \\
\text { distension }\end{array}$ \\
\hline 3 & eccentric & long bone metaphysis & variable lucency \\
\hline 4 & subperiosteal & long bone diaphysis & periosteal elevation \\
\hline 5 & $\begin{array}{l}\text { subperiosteal } \\
\text { expansive }\end{array}$ & cancellous bone & periosteal elevation with cortical destruction \\
\hline
\end{tabular}

\section{TABLE 1: Abbreviated Campanacci classification criteria of aneurysmal bone cyst types}

Per the Campanacci criteria, our case exemplifies a Type $1 \mathrm{ABC}$. This type of $\mathrm{ABC}$ makes up approximately $23 \%$ of $\mathrm{ABCs}$ and curettage through a boney window, as completed in our case, is the recommended treatment [7]. Today, the Campanacci criteria remain a useful method of categorizing $\mathrm{ABC}$ subtypes and their respective treatments.

Making the correct diagnosis is vitally important for the management of an $\mathrm{ABC}$. The most important alternative diagnosis that one must consider is telangiectatic osteosarcoma (TOC). TOCs are malignant primary bone neoplasms that are highly vascularized, lacking the expected solid osteoid production characteristic of typical osteosarcomas [8]. TOC presents as multiple cystic compartments on imaging, with fluid levels making it easy to confuse with an ABC [9]. The age of presentation between these two conditions is also similar, further complicating differentiation. Careful diagnosis is vital, as the treatment of TOC is based on that of osteosarcomas [10].

Radiographically, TOCs tend to grow more rapidly and invade nearby structures more consistently than ABCs. producing a soft tissue mass [11-12]. However, these basic radiographic tendencies can be insufficient for reliable diagnosis via imaging alone. Therefore, a biopsy is performed for further differentiation. The primary means of differentiating these two tumors histologically is the presence of cellular atypia. TOC is characterized by "highly atypical stromal cells with hyperchromasia, nuclear pleomorphism, and atypical mitoses" [11]. ABCs lack these malignant changes, making their differentiation possible under the microscope. 
There are a variety of approaches to the management of ABCs, ranging from medical therapy alone to curettage with grafting and even wide resection. Each method, with its respective risks and outcomes, presents patients and providers with the question of effective tumor management vs total morbidity and mortality. As detailed by Schreuder et al., wide resection vs curettage and bone grafting was associated with a recurrence rate of $0 \%$ vs approximately $31 \%$, respectively; however, wide resection was associated with a greater need for reconstructive surgery and its associated increased morbidity [13]. Because of this, it is reasonable to accept the relatively higher tumor recurrence rate associated with curettage and bone grafting alone as compared to wide resection based on the goal of a more rapid return to a functional baseline as demonstrated in our clinical case.

\section{Conclusions}

ABCs can present a diagnostic challenge even to the most skilled practitioner. Lesions of the distal femur can present as pathologic fractures and must be differentiated from the more ominous TOC. Once a diagnosis is established, the Campanacci criteria remain a useful starting point for lesion categorization and basic management. Treatment options are many, but curettage with bone grafting provides an acceptable compromise between tumor-free survival and treatment morbidity and mortality.

\section{Additional Information}

\section{Disclosures}

Human subjects: Consent was obtained by all participants in this study. Conflicts of interest: In compliance with the ICMJE uniform disclosure form, all authors declare the following: Payment/services info: All authors have declared that no financial support was received from any organization for the submitted work. Financial relationships: All authors have declared that they have no financial relationships at present or within the previous three years with any organizations that might have an interest in the submitted work. Other relationships: All authors have declared that there are no other relationships or activities that could appear to have influenced the submitted work.

\section{References}

1. Lawson C, Dormans JP: Benign pediatric bone tumors. Pediatr Clin North Am. 1996, 43:949966. 10.1016/s0031-3955(05)70444-2

2. Hakim DN, Pelly T, Kulendran M, Caris JA: Benign tumours of the bone: a review . J Bone Oncol. 2015, 2:37-41. 10.1016/j.jbo.2015.02.001

3. Mascard E, Gomez-Brouchet A, Lambot K: Bone cysts: unicameral and aneurysmal bone cyst . Orthop Traumatol Surg Res. 2015, 101:119-127. 10.1016/j.otsr.2014.06.031

4. Ibrahim T, Howard A, Murnaghan M, Hopyan S: Percutaneous curettage and suction for pediatric extremity aneurysmal bone cysts. J Pediatr Orthop. 2012, 32:842-847. 10.1097/bpo.0b013e31825d3619

5. Skubitz K, Peltola J, Santos E, Cheng E: Response of aneurysmal bone cyst to denosumab . Spine. 2015, 40:E1201-E1204. 10.1097/BRS.0000000000001027

6. Szendroi M, Cser I, Konya A, Renyi-Vamo A: Aneurysmal bone cyst. A review of 52 primary and 16 secondary cases. Arch Orthop Traum Su. 1992, 111:318-322. 10.1007/bf00420058

7. Campanacci M, Capanna R, Picci P: Unicameral and aneurysmal bone cysts. Clin Orthop Relat Res. 1986, 204:25-36. 10.1097/00003086-198603000-00004

8. Fletcher CDM: The evolving classification of soft tissue tumours - an update based on the new 2013 WHO classification 64, no. Histopathology. 2013, 64:2-11. 10.1111/his.12267

9. van Unnik JAM: Classification and grading of soft-tissue sarcomas. Hematol Oncol Clin North Am. 1995, 9:677-700. 10.1016/s0889-8588(18)30091-1

10. Angelini A, Mavrogenis AF, Trovarelli G, Ferrari S, Picci P, Ruggieri P: Telangiectatic osteosarcoma: a review of 87 cases. J Cancer Res Clin Oncol. 2016, 142:2197-2207. 


\section{Cureus}

10.1007/s00432-016-2210-8

11. Sangle NA, Layfield LJ: Telangiectatic osteosarcoma. Arch Pathol Lab Med. 2012, 136:572576. 10.5858/arpa.2011-0204-RS

12. Murphey MD, wan Jaovisidha S, Temple HT, Gannon FH, Jelinek JS, Malawer MM:

Telangiectatic osteosarcoma: radiologic-pathologic comparison. Radiology. 2003, 229:545553. 10.1148/radiol.2292021130

13. Schreuder HW, Veth RP, Pruszczynski M, Lemmens JA, Koops HS, Molenaar WM: Aneurysmal bone cysts treated by curettage, cryotherapy and bone grafting. J Bone Joint Surg Br. 1997, 79:20-25. 